\title{
An improved cosmological bound on the thermal axion mass
}

\author{
Alessandro Melchiorri ${ }^{1}$, Olga Mena ${ }^{1}$ and Anže Slosar ${ }^{2,3}$ \\ ${ }^{1}$ INFN Sez. di Roma, Dipartimento di Fisica, Università di Roma "La Sapienza", P.le A. Moro, 5, I-00185 Roma, Italy \\ ${ }^{2}$ Astrophysics, Denys Wilkinson Building, University of Oxford, Keble Road, OX3RH1, Oxford, UK and \\ ${ }^{3}$ Faculty of Mathematics and Physics, University of Ljubljana, Slovenia
}

(Dated: October 26, 2018)

\begin{abstract}
Relic thermal axions could play the role of an extra hot dark matter component in cosmological structure formation theories. By combining the most recent observational data we improve previous cosmological bounds on the axion mass $m_{a}$ in the so-called hadronic axion window. We obtain a limit on the axion mass $m_{a}<0.42 \mathrm{eV}$ at the $95 \%$ c.l. $\left(m_{a}<0.72 \mathrm{eV}\right.$ at the $99 \%$ c.l. $)$. A novel aspect of the analysis presented here is the inclusion of massive neutrinos and how they may affect the bound on the axion mass. If neutrino masses belong to an inverted hierarchy scheme, for example, the above constraint is improved to $m_{a}<0.38 \mathrm{eV}$ at the $95 \%$ c.l. $\left(m_{a}<0.67 \mathrm{eV}\right.$ at the $99 \%$ c.l.). Future data from experiments as CAST will provide a direct test of the cosmological bound.
\end{abstract}

PACS numbers:

\section{INTRODUCTION}

Recent Cosmic Microwave Background and Large Scale Structure surveys such as WMAP and SDSS have open the possibility of constraining fundamental physics with cosmology (see e.g. [1, 2]). Important upper limits on neutrino masses and energy densities, for example, have been obtained which are in some cases one order of magnitude better than the corresponding laboratory constraints $([2,3,4,5])$ or competitive with big bang nucleosynthesis constraints $(\underline{6}])$.

The cosmological limits are model dependent and therefore rely on the assumption of a theoretical model of structure formation that, even if in agreement with current data, may need further key ingredients to explain mysteries and inconsistencies such as dark energy. Moreover, for some datasets, the relevance of systematics is still matter of debate.

However, future laboratory experiments will certainly test the cosmological results. The overlap of cosmological and laboratory limits will open a new window of investigation and may provide evidence for new physics and/or improve our knowledge of systematics.

It is therefore timely to constrain fundamental physics with cosmology. In this paper we indeed move along one of those lines of investigation, providing new bounds on the thermal axion mass from cosmology. There are two possible ranges of axion masses $(\sim \mu \mathrm{eV}$ and $\sim \mathrm{eV})$ and, in principle, both could provide either a dominant or a sub-dominant dark matter component. Here we focus on thermal axions with masses of $\sim \mathrm{eV}$. For a recent revival of the cold dark matter scenario with axions of masses $\sim \mu \mathrm{eV}$, see Ref. [7]. New constraints on the thermal axion mass and couplings have recently been presented by the CAST experiment, which searchs for axion-like particles from the Sun which couple to photons [8]. While the axion mass region probed by the CAST experiment is one order of magnitude lower than the cosmological bound presented here, an overlap of the two results is clearly around the corner.

Let us remind the origin of the axions. Quantum Chromodynamics (QCD) respects CP symmetry, despite the existence of a natural, four dimensional, Lorentz and gauge invariant operator which badly violates CP. The former extra $\mathrm{CP}$ violating-term gives rise to physical observables, namely, to a non-vanishing neutron dipole moment, $d_{n}$. The existing tight bound $\left|d_{n}\right|<$ $3 \times 10^{-26} e \mathrm{~cm}[9]$ requires the $\mathrm{CP}$ term contribution to be very small. Why are $\mathrm{CP}$ violating effects so small in QCD? Why is CP not broken in QCD? This is known as the strong $\mathrm{CP}$ problem. The most convincing, and elegant, solution to the strong CP problem was provided by Peccei and Quinn [10], by adding a new global $U(1)_{P Q}$ symmetry. This symmetry is spontaneously broken at a large energy scale $f_{a}$, generating a new spinless particle, the axion, allowing for a dynamical restoration of the $\mathrm{CP}$ symmetry. Axions are the pseudo Nambu-Goldstone bosons of the broken $U(1)_{P Q}$ symmetry [11, 12] and may be copiously produced in the early universe, either thermally [13] or non-thermally [14], providing a possible (sub)dominant (hot) dark matter candidate. The axion mass and couplings are inversely proportional to the axion coupling constant $f_{a}$

$$
m_{a}=\frac{f_{\pi} m_{\pi}}{f_{a}} \frac{\sqrt{R}}{1+R}=0.6 \mathrm{eV} \frac{10^{7} \mathrm{GeV}}{f_{a}},
$$

where $R=0.553 \pm 0.043$ is the up-to-down quark masses ratio [15] and $f_{\pi}=93 \mathrm{MeV}$ is the pion decay constant. In principle, axions can interact with photons, electrons and hadrons. If axions couple to photons and electrons, the simplest bound comes from an energy loss argument. The axions produced in a star escape carrying away energy, producing anomalous stellar observables, see Refs. [16, 17, 18] for a review. However, in practice, axion interactions are model dependent. Here we focus on hadronic axion models such as the KSVZ 
model [19, 20], in which there is no tree level interaction between axions and leptons and the axion-photon coupling could accidentally be negligibly small. Hannestad et al 21] have recently found an upper limit on the hadronic axion mass $m_{a}<1.05 \mathrm{eV}$ (95\% CL), which translates into $f_{a}>5.7 \times 10^{6} \mathrm{GeV}$. In this letter, we reinforce the former limit by means of an updated analysis, using a broad set of the most recent available cosmological data, and allowing for two possible hot dark matter components: neutrinos and axions.

\section{THE HADRONIC AXION MODEL}

Among axion couplings with hadrons, those of interest for us are the axion-nucleon couplings $\mathcal{L}_{a N}$, responsible for the processes $N+N \leftrightarrow N+N+a$ and $N+\pi \leftrightarrow$ $N+a$, and the axion-pion couplings $\mathcal{L}_{a \pi}$, responsible for $a+\pi \leftrightarrow \pi+\pi$. In practice, nucleons are so rare in the early universe respect to pions, that only the axion-pion interaction will be relevant for thermalization purposes. The lagrangian reads [22]

$\mathcal{L}_{a \pi}=C_{a \pi} \frac{\partial_{\mu} a}{f_{a} f_{\pi}}\left(\pi^{0} \pi^{+} \partial_{\mu} \pi^{-}+\pi^{0} \pi^{-} \partial_{\mu} \pi^{+}-2 \pi^{+} \pi^{-} \partial_{\mu} \pi^{0}\right)$

where

$$
C_{a \pi}=\frac{1-R}{3(1+R)}
$$

is the axion-pion coupling constant [22]. The most stringent limits on the axion-nucleon coupling in hadronic axion models, $g_{a N}=C_{N} m_{N} / f_{a}$, are those coming from SN 1987A neutrino data. If axions couple to nucleons strongly, the supernova cooling process is modified, distorting both the measured neutrino flux and the duration time of the neutrino burst emitted. The limit in the axion-nucleon coupling $g_{a N}$, assuming that the modeldependent parameter $C_{N} \simeq \mathcal{O}(1)$, translates into an axion decay constant $f_{a} \lesssim$ few $\times 10^{-6} \mathrm{GeV}$ [23]. Even if axion emission does not affect the SN cooling, if $g_{a N}$ is strong enough, the axion flux may excite ${ }^{16} \mathrm{O} \mathrm{nu}-$ clei in water Cherenkov detectors. The absence of a large signal from radiative decays of excited ${ }^{16} O^{\star}$ nuclei in the Kamiokande experiment provides a lower limit $f_{a} \gtrsim 3 \times 10^{5} \mathrm{GeV}[24]$. In summary, hadronic axions with the decay constant $f_{a}$ around $10^{6} \mathrm{GeV}$, i.e. $m_{a} \sim \mathrm{eV}$, can escape from all astrophysical and laboratory constraints known so far, suggesting an ideal hot dark matter candidate, within the mixed hot dark matter scenario [25].

\section{AXION DECOUPLING}

Axions will remain in thermal equilibrium until the expansion rate of the universe, given by the Hubble pa- rameter $H(T)$, becomes larger than their thermally averaged interaction rate. To compute the axion decoupling temperature $T_{D}$ we follow the usual freeze out condition

$$
\Gamma\left(T_{D}\right)=H\left(T_{D}\right) .
$$

The axion interaction rate $\Gamma$ is given by [22]

$$
\Gamma=n_{a}^{-1} \sum_{i, j} n_{i} n_{j}\left\langle\sigma_{i j} v\right\rangle,
$$

where $n_{a}=\left(\zeta_{3} / \pi^{2}\right) T^{3}$ is the number density for axions in thermal equilibrium, and the sum extends to all production processes involving as initial states the particles $i$ and $j$, which are in equilibrium at $T_{D}$. We will assume that the axion decay constant $f_{a}$ is sufficiently small to ensure that axions decouple from the thermal plasma after the QCD transition epoch at $T=T_{Q C D} \simeq 200 \mathrm{MeV}$ $\left(f_{a} \lesssim 4 \times 10^{7} \mathrm{GeV}\right.$, i.e., $\left.m_{a} \gtrsim 0.14 \mathrm{eV}\right)$. Consequently, we do not have to consider axion interactions with the quarks and gluons before the QCD phase transition and the dominant processes contributing to the thermally averaged cross section in Eq. (5) will be $\pi^{0} \pi^{ \pm} \rightarrow a \pi^{ \pm}$and $\pi^{+} \pi^{-} \rightarrow a \pi^{0}$, see the interaction lagrangian, Eq. (2). (2)We follow here the computation carried out by Chang and Choi 22] for the average rate $\pi+\pi \rightarrow \pi+a$ :

$$
\Gamma=\frac{3}{1024 \pi^{5}} \frac{1}{f_{a}^{2} f_{\pi}^{2}} C_{a \pi}^{2} I,
$$

where

$$
\begin{aligned}
I & =n_{a}^{-1} T^{8} \int d x_{1} d x_{2} \frac{x_{1}^{2} x_{2}^{2}}{y_{1} y_{2}} f\left(y_{1}\right) f\left(y_{2}\right) \\
& \times \int_{-1}^{1} d \omega \frac{\left(s-m_{\pi}^{2}\right)^{3}\left(5 s-2 m_{\pi}^{2}\right)}{s^{2} T^{4}} .
\end{aligned}
$$

Here $f(y)=1 /\left(e^{y}-1\right)$ denotes the pion distribution function, $x_{i}=\left|\vec{p}_{i}\right| / T, y_{i}=E_{i} / T(i=1,2), s=2\left(m_{\pi}^{2}+\right.$ $\left.T^{2}\left(y_{1} y_{2}-x_{1} x_{2} \omega\right)\right)$, and we assume a common mass for the charged and neutral pions, $m_{\pi}=138 \mathrm{MeV}$.

The RHS in Eq. (4) contains the Hubble expansion rate, related to the energy density of the universe via the Friedmann equation [14]:

$$
H(T)=\sqrt{\frac{4 \pi^{3}}{45} g_{\star}(T)} \frac{T^{2}}{M_{p l}},
$$

where $M_{p l}$ is the Planck mass. We have computed, for temperatures $T$ in the range $1 \mathrm{MeV}<T<200 \mathrm{MeV}$, i.e. between $\mathrm{BBN}$ and the $\mathrm{QCD}$ phase transition eras, the number of relativistic degrees of freedom $g_{\star}(T)$, according to Ref. [14]. We neglect the axion contribution to $g_{\star}$ for simplicity. After resolving the freeze out equation Eq. (4), we obtain the axion decoupling temperature $T_{D}$ versus the axion mass $m_{a}$ (or, equivalently, versus the axion decay constant $f_{a}$ ). From the axion decoupling temperature, we can compute the current axion 
number density, related to the present photon density $n_{\gamma}=410.5 \pm 0.5 \mathrm{~cm}^{-3}[23]$ via

$$
n_{a}=\frac{g_{\star S}\left(T_{0}\right)}{g_{\star S}\left(T_{D}\right)} \times \frac{n_{\gamma}}{2},
$$

where $g_{\star S}$ refers to the number of entropic degrees of freedom. Before electron-positron annihilation at temperatures $\sim \mathrm{eV}$, the number of entropic degrees of freedom is $g_{\star S}=g_{\star}$, since all relativistic particles are at the same temperature. At the current temperature, $g_{\star S}\left(T_{0}\right)=3.91[14]$.

\section{COSMOLOGICAL CONSTRAINTS}

As now common practice in the literature we derive our constraints by analyzing Monte Carlo Markov Chain of cosmological models. For this purpose we use a modified version of the publicly available Cosmo-MCMC package cosmomc 26] with a convergence diagnostics done through the Gelman and Rubin statistic. We sample the following eight-dimensional set of cosmological parameters, adopting flat priors on them: the baryon and Cold Dark Matter densities, $\omega_{b}=\Omega_{b} h^{2}$ and $\omega_{c}=\Omega_{c} h^{2}$, the ratio of the sound horizon to the angular diameter distance at decoupling, $\theta_{s}$, the scalar spectral index $n_{S}$, the overall normalization of the spectrum $A$ at $k=0.05 \mathrm{Mpc}^{-1}$, the optical depth to reionization, $\tau$, the energy density in massive neutrinos

$$
\Omega_{\nu} h^{2}=\frac{\sum m_{\nu}}{92.5 \mathrm{eV}}
$$

and the energy density in the thermal axions:

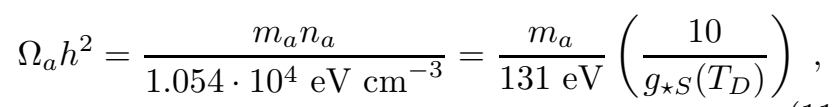

where we have used Eq. (9). For instance, for the hadronic axion upper mass bound quoted in Ref. 21], i.e. $m_{a} \sim 1.05 \mathrm{eV}$, the axion decouples at $T_{D} \sim 64 \mathrm{MeV}$, at which $g_{\star S}\left(T_{D}\right) \simeq 15.24$ and $\Omega_{a} h^{2} \simeq 0.0053$.

We consider a combination of cosmological data which includes the three-year WMAP data [1], the small-scale CMB measurements of CBI 27], VSA [28], ACBAR 29] and BOOMERANG-2k2 30]. In addition to the CMB data, we include the constraints on the real-space power spectrum of red luminous giant (LRG) galaxies from the fourth data release of the SLOAN galaxy redshift survey (SDSS) 31 and 2dF 32], and the Supernovae Legacy Survey data from [33]. Finally we include a prior on the Hubble parameter from the Hubble Space Telescope Key project [34] and the BBN prior in form of a Gaussian prior on $\Omega_{b} h^{2}$ (see e.g. [6]). We refer to this dataset as Conservative in the rest of the paper.

\begin{tabular}{ccc} 
dataset/prior & $m_{a}<$ & $\sum m_{\nu}<$ \\
\hline Conservative & $1.4 / 2.0 \mathrm{eV}$ & $0.55 / 0.9 \mathrm{eV}$ \\
\hline Conservative+LYA & $0.42 / 0.72 \mathrm{eV}$ & $0.20 / 0.37 \mathrm{eV}$ \\
$+\Sigma m_{\nu}>0.05 \mathrm{eV}$ & $0.41 / 0.71 \mathrm{eV}$ & $0.22 / 0.38 \mathrm{eV}$ \\
$+\Sigma m_{\nu}>0.1 \mathrm{eV}$ & $0.38 / 0.67 \mathrm{eV}$ & $0.25 / 0.44 \mathrm{eV}$ \\
\hline Conservative+LYA+BAO & $0.35 / 0.64 \mathrm{eV}$ & $0.18 / 0.31 \mathrm{eV}$
\end{tabular}

TABLE I: This figure shows the 95\%/99.9\% upper confidence limits on the marginalised posterior probabilities for axion and neutrino masses. See text for discussion.

In the second dataset we include constraints on the small scale linear power spectrum coming from Lyman- $\alpha$ analysis of SDSS quasar spectra [37, 38].

The main results of our analysis are reported in the Table I As can see, without assuming any prior on the neutrino mass, the mass of the thermal axion is found to be $m_{a}<0.42 \mathrm{eV}$ and the sum of the three active massive neutrinos $\sum m_{\nu}<0.20 \mathrm{eV}$, both at the $95 \%$ c.l., i.e $\Omega_{a} h^{2}<0.0014$ and $\Omega_{\nu} h^{2}<0.0018$. Therefore, the neutrino-axion (hot) dark matter contribution represents a small fraction $(\lesssim 2.5 \%)$ of the total CDM. Excluding from the analysis the constraints from BAO and Lyman$\alpha$ cosmological datasets the former limits translate into $m_{a}<1.4 \mathrm{eV}$ and $\sum m_{\nu}<0.55 \mathrm{eV}$. The inclusion of the Lyman- $\alpha$ data has an enormous impact on the analysis. In the same table we also consider the effect of adding Baryonic Acoustic Oscillations (BAO) data detected in the Luminous Red Galaxies (LRG) sample of the SDSS 36] to the data. Strictly speaking this is a statistically incorrect procedure as the correlations with SDSS LRG power spectrum are not well understood, but it gives the idea of the improvements that can be achieved by including BAO constraints.

In Fig. 1, where we present marginalized constraints on the $\sum m_{\nu}-m_{a}$ plane. There is a clear anti-correlation between the constraints on the thermal axion mass and the mass of the three active neutrinos. In other words, the cosmological data allow only for a very specific quantity of hot dark matter: if one increases the active neutrino mass, more hot dark matter is present in the model and the axion mass has to be smaller in order to fit the observations.

Figure 2 depicts the 95\% CL axion mass limits in the $m_{a^{-}} g_{a \gamma \gamma}$ (axion-to-photon coupling) plane. The limits should be within the region allowed by the KSVZ model. We have considered two possible scenarios, accordingly to neutrino oscillation data: normal hierarchy $\left(\sum m_{\nu} \gtrsim \sqrt{\left|\Delta m_{13}^{2}\right|} \gtrsim 0.05 \mathrm{eV}\right)$ and inverted hierarchy $\left(\sum m_{\nu} \gtrsim 2 \cdot \sqrt{\left|\Delta m_{13}^{2}\right|} \gtrsim 0.1 \mathrm{eV}\right)$, as well as the massless neutrino case. The $95 \%$ c.l. constraints that we obtain for the axion mass within the three possible scenarios mentioned above are $m_{a}<0.34,0.31$ and $0.34 \mathrm{eV}$, respectively, including both BAO and Lyman- $\alpha$ datasets. We found no significant difference between the normal hierarchy and the massless neutrino scenarios. If fu- 

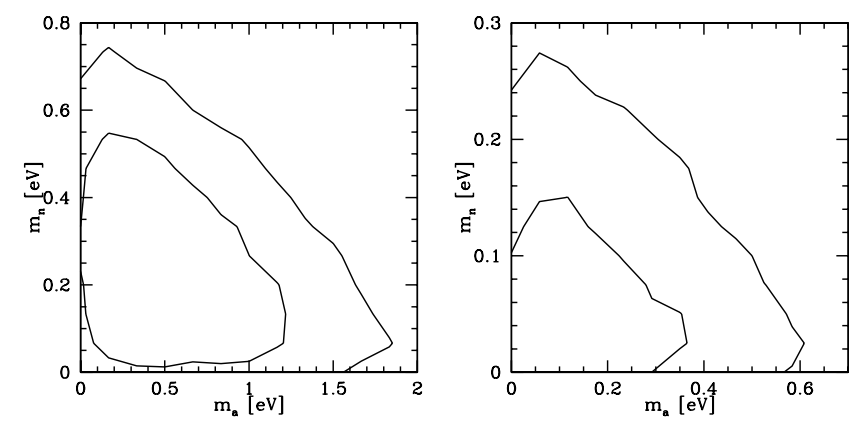

FIG. 1: Likelihood contour plot in the $\sum m_{\nu}-m_{a}$ plane showing the $68 \%$ and $95 \%$ c.l. from the conservative dataset (left panel) and from the complete dataset (right panel). Note different axes.

ture cosmological data [40] or direct terrestrial searches for neutrino masses, as the ones which will be carried out by the KATRIN experiment [41], improve the current limits on $\sum m_{\nu}$, one could obtain automatically a rather robust, independent, albeit indirect limit on the axion mass $m_{a}$. We depict in Fig. 2 the current $95 \%$ c.l. CAST limit for comparison [8]. The CAST experiment has been upgraded and in the near future it will explore QCD axions, that is, a range of axion masses up to about $1 \mathrm{eV}$. Cosmology-independent future limits on the axion mass are therefore extremely important, since they could provide a test of the cosmological constraint and be translated into a limit of the universe's hot dark matter fraction in the form of massive neutrinos.

\section{CONCLUSIONS}

We have presented an improved limit on the hadronic axion mass by combining the most recent available cosmological data. A novel content of this analysis is the addition of a hot dark matter component in the form of massive neutrinos. Interestingly, we have noticed an anticorrelation between the thermal axion mass and the mass of the three active neutrinos $\sum m_{\nu}$. This anti-correlation is due to the suppression induced on the small scale power spectra by both the relic axion and the massive neutrino free-streaming species. A larger (smaller) axion mass content can be traded by a smaller (larger) massive neutrino content. If the complete cosmological dataset is used, we find $m_{a}<0.35 \mathrm{eV}$ and $\sum m_{\nu}<0.17 \mathrm{eV}$ at the $95 \%$ c.l., implying that the fraction of (hot) dark matter in the form of massive thermal axions and neutrinos is only a few percent $(\lesssim 2.5 \%)$ of the total CDM content. The former limits get modified if priors on the neutrino or axion masses are imposed. Future cosmological and/or terrestrial searches for neutrino (axion) masses could therefore be translated into an improved and independent axion (neutrino) mass limit.

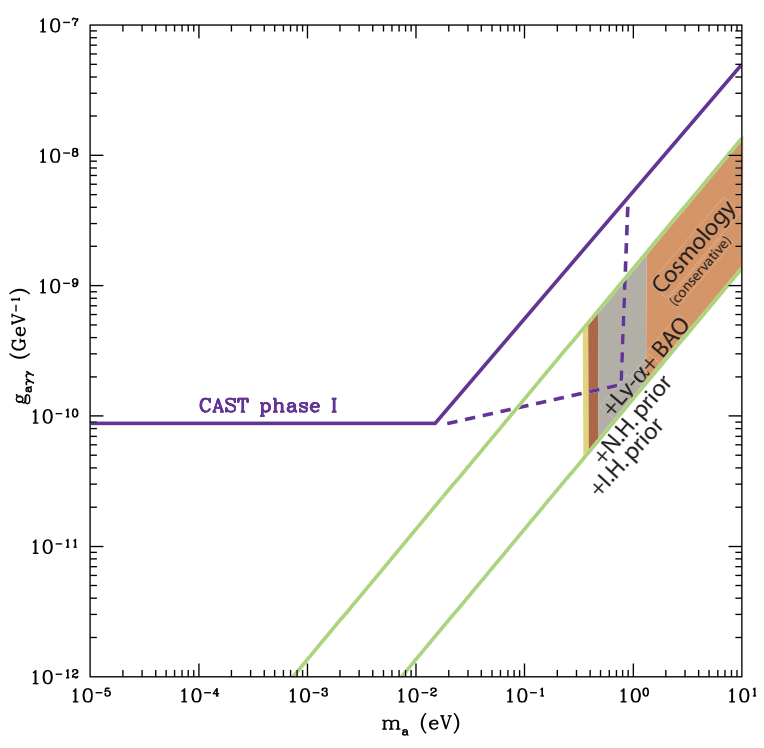

FIG. 2: $95 \%$ CL limits on the axion mass obtained in the conservative and full analysis (shaded regions), assuming three possible values of the sum of the neutrino masses in the $m_{a^{-}}$ $g_{a \gamma \gamma}$ plane. From right to left the region represent the exclusion limits assuming a prior $\sum m_{\nu}>0, \sum m_{\nu}>0.05 \mathrm{eV}$ (N.H.) and $\sum m_{\nu}>0.1 \mathrm{eV}$ (I.H). As a comparison, we show the recent results from the CAST experiment (blue contour) as well as the theoretical KSVZ parameter region (within the green lines), following Fig. 8 from Ref. [8], and the CAST prospects (blue dashed line) [39].

\section{ACKNOWLEDGMENTS}

It is a pleasure to thank Alessandro Mirizzi for useful discussions. The work of O. M is supported the European Programme "The Quest for Unification" contract MRTN-CT-2004-503369. Results were computed on the UK-CCC COSMOS supercomputer.

[1] Spergel D N et al., Wilkinson microwave anisotropy probe (WMAP) three year results: implications for cosmology, 2006 Preprint astro-ph/0603449.

[2] Seljak U, Slosar A and McDonald P, 2006 JCAP 0610 014 astro-ph/0604335.

[3] G. L. Fogli et al., Phys. Rev. D 75 (2007) 053001 arXiv:hep-ph/0608060; G. L. Fogli, et al., Phys. Rev. D 70 (2004) 113003 arXiv:hep-ph/0408045.

[4] J. Lesgourgues and S. Pastor, Phys. Rept. 429 (2006) 307 arXiv:astro-ph/0603494.

[5] S. Dodelson, A. Melchiorri and A. Slosar, Phys. Rev. Lett. 97 (2006) 04301 arXiv:astro-ph/0511500.

[6] J. Hamann, S. Hannestad, G. G. Raffelt and Y. Y. Y. Wong, arXiv:0705.0440 [astro-ph]; G. Mangano, A. Melchiorri, O. Mena, G. Miele and A. Slosar, JCAP 0703 (2007) 006 arXiv:astro-ph/0612150; S. H. Hansen, et al., Phys. Rev. D 65 (2002) 023511 
arXiv:astro-ph/0105385.

[7] M. R. Buckley and H. Murayama, arXiv:0705.0542 [hep$\mathrm{ph}]$.

[8] S. Andriamonje et al. [CAST Collaboration], JCAP 0702, 010 (2007) arXiv:hep-ex/0702006.

[9] C. A. Baker et al., Phys. Rev. Lett. 97, 131801 (2006) arXiv:hep-ex/0602020.

[10] R. D. Peccei and H. R. Quinn, Phys. Rev. Lett. 38, 1440 (1977); R. D. Peccei and H. R. Quinn, Phys. Rev. D 16, 1791 (1977).

[11] S. Weinberg, Phys. Rev. Lett. 40, 223 (1978).

[12] F. Wilczek, Phys. Rev. Lett. 40, 279 (1978).

[13] M. S. Turner, Phys. Rev. Lett. 59, 2489 (1987) [Erratumibid. 60, 1101 (1988)].

[14] E. W. Kolb and M. S. Turner, "The Early Universe", Addison Wesley (1990).

[15] H. Leutwyler, Phys. Lett. B 378, 313 (1996)

[16] G. Raffelt, "Stars as Laboratories for Fundamental Physics: The Astrophysics of Neutrinos, Axions, and Other Weakly Interacting Particles", University of Chicago Press (1996).

[17] G. G. Raffelt, Ann. Rev. Nucl. Part. Sci. 49, 163 (1999) arXiv:hep-ph/9903472.

[18] G. G. Raffelt, arXiv:hep-ph/0611350.

[19] J. E. Kim, Phys. Rev. Lett. 43, 103 (1979).

[20] M. A. Shifman, A. I. Vainshtein and V. I. Zakharov, Nucl. Phys. B 166, 493 (1980).

[21] S. Hannestad, A. Mirizzi and G. Raffelt, JCAP 0507, 002 (2005) arXiv:hep-ph/0504059.

[22] S. Chang and K. Choi, Phys. Lett. B 316, 51 (1993)

[23] W. M. Yao et al. [Particle Data Group], J. Phys. G 33, 1 (2006).

[24] J. Engel, D. Seckel and A. C. Hayes, Phys. Rev. Lett. 65, 960 (1990).

[25] T. Moroi and H. Murayama, Phys. Lett. B 440, 69 (1998)
arXiv:hep-ph/9804291.

[26] Lewis A and Bridle S, 2002 Phys. Rev. D 66103511 astro-ph/0205436.

[27] Readhead A C S et al., 2004 ApJ $\mathbf{6 0 9} 498$ astro-ph/0402359.

[28] Dickinson C et al., 2004 MNRAS $353 \quad 732$ astro-ph/0402498.

[29] Kuo C L et al., 2002 American Astronomical Society Meeting Vol. 201.

[30] MacTavish C J et al., 2006 Astrophys. J. 647799 astro-ph/0507503.

[31] Tegmark M et al., 2004 ApJ 606702 astro-ph/0310725.

[32] Cole S et al. [The 2dFGRS Collaboration], 2005 Mon. Not. Roy. Astron. Soc. 362505 astro-ph/0501174.

[33] Astier P et al., 2006 Astron. Astrophys. 44731 astro-ph/0510447.

[34] Freedman W L et al., 2001 Astrophys. J. 55347 astro-ph/0012376.

[35] Tegmark M et al., Cosmological Constraints from the SDSS Luminous Red Galaxies, 2006 Preprint astro-ph/0608632

[36] Eisenstein D J et al., 2005 Astrophys. J. 633560 astro-ph/0501171.

[37] McDonald P et al., 2006 Astrophys. J. Suppl. 16380 astro-ph/0405013.

[38] McDonald P et al., 2005 Astrophys. J. 635761 astro-ph/0407377.

[39] K. Zioutas et al., Nucl. Instrum. Meth. A 425, 480 (1999) arXiv:astro-ph/9801176.

[40] S. Hannestad and Y. Y. Y. Wong, arXiv:astro-ph/0703031

[41] G. Drexlin [KATRIN Collaboration], Nucl. Phys. Proc. Suppl. 145 (2005) 263. 